\title{
Regulation of Gastric Emptying in Humans by Cholecystokinin
}

\author{
Rodger A. Liddle, Eugene T. Morita, Carol K. Conrad, and John A. Williams
}

Cell Biology Laboratory and Department of Nuclear Medicine, Mt. Zion Hospital and Medical Center, San Francisco, California 94120; and Departments of Medicine and Physiology, University of California, San Francisco, California 94143

\begin{abstract}
In the present study we used a bioassay system for measuring plasma cholecystokinin (CCK) to evaluate whether CCK has a physiologic role in regulating gastric emptying in humans. Plasma CCK levels and gastric emptying after ingestion of a mixed liquid meal were determined in five normal male volunteers. Fasting CCK levels averaged $0.8 \pm 0.1 \mathrm{pM}$ and increased to $6.5 \pm 1.0 \mathrm{pM}$ within 10 min of drinking the mixed meal. CCK levels remained elevated for up to $90 \mathrm{~min}$. Gastric emptying after a meal was slow; at the end of the $90 \mathrm{~min} 68 \%$ of the original volume remained in the stomach. The rate of gastric emptying of water was then measured in the same individuals with a simultaneous infusion of either saline, or one of two doses of CCK (12 pmol/ $\mathrm{kg}$ per $\mathrm{h}$ and $24 \mathrm{pmol} / \mathrm{kg}$ per $\mathrm{h}$ ). With the saline infusion, plasma CCK levels did not increase above basal and gastric contents emptied rapidly. At the end of 90 min only $7 \%$ of the original volume remained in the stomach. The lower dose of CCK resulted in a plasma level of $3.4 \mathrm{pM}$ which both reproduced the average postprandial plasma level and caused a significant delay in gastric emptying. The higher dose of CCK achieved plasma levels of 8 pM and resulted in a delay in gastric emptying that was similar to that seen with the mixed meal. Since exogenous CCK at concentrations which occur postprandially delays gastric emptying, we conclude that $\mathrm{CCK}$ is a physiologic regulator of gastric emptying.
\end{abstract}

\section{Introduction}

The physiologic mechanisms which regulate emptying of food from the stomach are complex and their interrelationships are incompletely understood. Intrinsic properties of ingested food, such as volume, osmolality, $\mathrm{pH}$, temperature, and ion and nutrient content have been demonstrated to affect the rate at which liquids empty from the stomach (1-6). It is unknown, however, to what extent nervous and hormonal influences may play a role in mediating such effects. Liquids with nutrient value, particularly fats and protein, empty from the stomach at a rate that is slower than either the emptying of water or saline $(7,8)$. This finding is of particular interest because these foods are the pri-

This work was presented in part at the American Gastroenterological Association Meeting in New York, NY on 15 May 1985 and published in abstract form, 1985. Gastroenterology. 88:1476.

Address correspondence to Dr. Liddle, Cell Biology Laboratory, Mount Zion Hospital, P.O. Box 7921, 1600 Divisadero, San Francisco, CA 94120.

Received for publication 18 July 1985.

J. Clin. Invest.

(c) The American Society for Clinical Investigation, Inc.

0021-9738/86/03/0992/05 \$1.00

Volume 77, March 1986, 992-996 mary stimulants of cholecystokinin $(\mathrm{CCK})^{1}$ release from the intestine (9). It has been demonstrated in experimental animals and humans that $\mathrm{CCK}$, at pharmacologic doses, inhibits gastric emptying (10-15). However, because of prior difficulties in measuring plasma CCK levels, it was unclear whether this effect of CCK on gastric emptying could occur at physiologic concentrations of this hormone.

We have recently developed a bioassay for measuring plasma CCK levels in humans $(9,16)$. It is now possible, therefore, to determine the levels of CCK that occur in response to feeding. In the present study we measured CCK levels after the ingestion of a mixed meal. In addition, we measured CCK levels after the ingestion of water and the simultaneous infusion of a physiologic concentration of CCK. In studies with both the mixed meal and water the rate of gastric emptying was measured by radionuclide scintigraphic scanning. These experiments indicate that CCK has a physiologic role in regulating gastric emptying in humans.

\section{Methods}

Bioassay of CCK. Plasma CCK concentrations were measured by a specific bioassay as previously described $(9,16)$. This assay is based on the ability of CCK to stimulate amylase release from isolated rat pancreatic acini. Briefly, plasma was extracted and concentrated by adsorption onto octadecylsilylsilica cartridges (Sep-Pak; Waters Associates, Millipore Corp., Milford, MA). CCK was then eluted with $1 \mathrm{ml}$ of $80 \%$ ethanol/ $0.2 \%$ trifluoroacetic acid and the extracts were dried under nitrogen. Plasma extracts were then incubated with isolated rat pancreatic acini for $30 \mathrm{~min}$ at $37^{\circ} \mathrm{C}$ and the amylase released into the incubation medium assayed using Procion yellow-coupled starch as substrate. Amylase expressed as percent of total amylase content was compared with a doseresponse curve for CCK-8. With this method plasma CCK levels as low as $0.2 \mathrm{pM}$ could be detected. Intraassay and interassay coefficients of variation were 7.4 and $10.4 \%$, respectively.

Feeding, CCK infusion, and measurement of gastric emptying. The subjects for all studies were healthy male volunteers between 26 and 35 yr of age. Subjects underwent an overnight (12-15 h) fast before each study performed. All experiments were performed between 8 and 11 a.m. Blood samples were drawn from an indwelling 19-gauge butterfly catheter in the antecubital fossa during the 2 -h course of study. Infusions of either saline or CCK-8 were administered intravenously in the opposite arm.

These subjects were administered orally $400 \mathrm{ml}$ of either a mixed liquid meal or water to each of which $100 \mathrm{uCi}$ of technetium $\left(\mathrm{Tc}^{99 \mathrm{~m}}\right)$ sulfur colloid had been added. The mixed meal was made of Carnation instant breakfast supplement, one egg, and half and half milk and cream, totaling $1.5 \mathrm{cal} / \mathrm{ml}$ and consisting of $40 \%$ fat, $20 \%$ protein, and $40 \%$ carbohydrate. The osmolality of the mixed meal was $600 \mathrm{mOsm}$. Each meal was consumed over a 1 -min period. Blood was drawn at various times for CCK level determinations for up to $90 \mathrm{~min}$ after feeding.

On four separate days the same subjects were studied under the following conditions: $(a)$ ingestion of the mixed liquid meal, $(b)$ ingestion of water with simultaneous intravenous infusion of saline, $(c)$ ingestion of water with simultaneous infusion of CCK-8 at a rate of $12 \mathrm{pmol} / \mathrm{kg}$

1. Abbreviations used in this paper: $\mathrm{CCK}$, cholecystokinin; $\mathrm{Tc}^{99 \mathrm{~m}}$, technetium $99 \mathrm{~m}$. 
per $\mathrm{h}$, and $(d)$ ingestion of water with infusion of CCK-8 at $24 \mathrm{pmol} / \mathrm{kg}$ per $h$. For these studies CCK-8 (Sincalide; E. R. Squibb and Sons, Inc., Princeton, N. J.) was diluted to appropriate concentrations in a total volume of $20 \mathrm{ml}$ normal saline. By use of a pump (Harvard Apparatus Co., Inc., The Ealing Corp., S. Natick, MA), CCK was infused through an indwelling butterfly catheter in an antecubital vein. The actual infusion rate was determined by measuring the $\mathrm{CCK}$ concentration of the infusate ejected from the delivery system. This measurement corrects for losses of CCK by adsorption of syringes and intravenous tubing. The actual infusion rate was $46 \%$ of the rate calculated from the amount of CCK added, indicating that approximately one-half of the CCK had been lost onto tubing and syringes. No adverse effects from the CCK infusion were reported by any subjects. All meals were $400 \mathrm{ml}$ vol administered at room temperature.

Determinations of gastric emptying were made by gamma scintigraphy of the abdomen $(17,18)$. A gamma camera interfaced to an ADAC 3350 computer (Analytical Development Assoc. Corp., San Jose, CA) and fitted with a high resolution, low energy parallel hole collimator was used for imaging. Subjects remained seated between counts but stood in front of the camera for counts taken every $5 \mathrm{~min}$ for the first $30 \mathrm{~min}$ of the test, and every $15 \mathrm{~min}$ thereafter. Although data was collected in both the anterior and posterior projections, only the anterior views were used in calculating the gastric emptying since the geometric means of the anterior and posterior projections were very similar to those of the anterior views alone (18). Data was stored on computer discs for subsequent analysis. Using the computer display, the gastric regions were outlined, excluding the small intestine. For each time point, the total radioactivity in the region of interest was summated. Background counts were substracted and corrections for radionuclide decay were calculated to yield final values for residual radioactivity remaining in the stomach. Background counts were small with respect to the total counts. Counts in the gastric region were expressed as a percentage of the total counts in the whole field in the first five postcibal minutes. The stomach and whole body radiation doses were calculated to be 11 and 1.7 millirads, respectively, for each study (calculations by Mr. Phillip Purser, Mt. Zion Hospital and Medical Center, San Francisco, CA).

This study was approved by the Committee on the Protection of Human Subjects of Mt. Zion Hospital. Written informed consent was obtained from each subject.

Statistical analysis. Values are expressed as the mean \pm 1 SEM. Comparison of responses were made by analysis of variance with repeated measures (19). Post-hoc analysis of the difference between points was carried out by means of the Newman-Keuls test. Comparisons between model parameters for gastric emptying studies were made by Student's $t$ test. Differences with a $P$ value of $<0.05$ were considered significant.

\section{Results}

Plasma CCK response to feeding. The stimulatory effect of food on plasma CCK levels was studied in five normal male volunteers (Fig. 1 $A$ ) Each subject was fed $400 \mathrm{ml}$ of a mixed liquid meal labeled with $\mathrm{Tc}^{99 \mathrm{~m}}$-sulfur colloid and plasma CCK levels measured for up to $90 \mathrm{~min}$. Fasting levels of CCK averaged $0.8 \pm 0.1$ pM CCK-8 equivalents and rose promptly to $6.5 \pm 1.0 \mathrm{pM}$ within $10 \mathrm{~min}$ of feeding. CCK levels declined over the next $20 \mathrm{~min}$ to $3 \mathrm{pM}$ and remained elevated for the 90 -min period of study.

Gastric emptying of a liquid meal. To determine the rate at which the radiolabeled meal emptied from the stomach, scintigraphic scanning over the area of the stomach was conducted for 90 min postcibally (Fig. $1 \mathrm{~B}$ ). The rate of gastric emptying was slow with $68.3 \%$ of the initial volume remaining in the stomach after $90 \mathrm{~min}$.

Plasma CCK levels produced by infusion of exogenous CCK. To establish whether CCK has a physiologic role in regulating gastric emptying, CCK was infused into subjects to reproduce normally occurring postprandial CCK levels and the rate of gas-
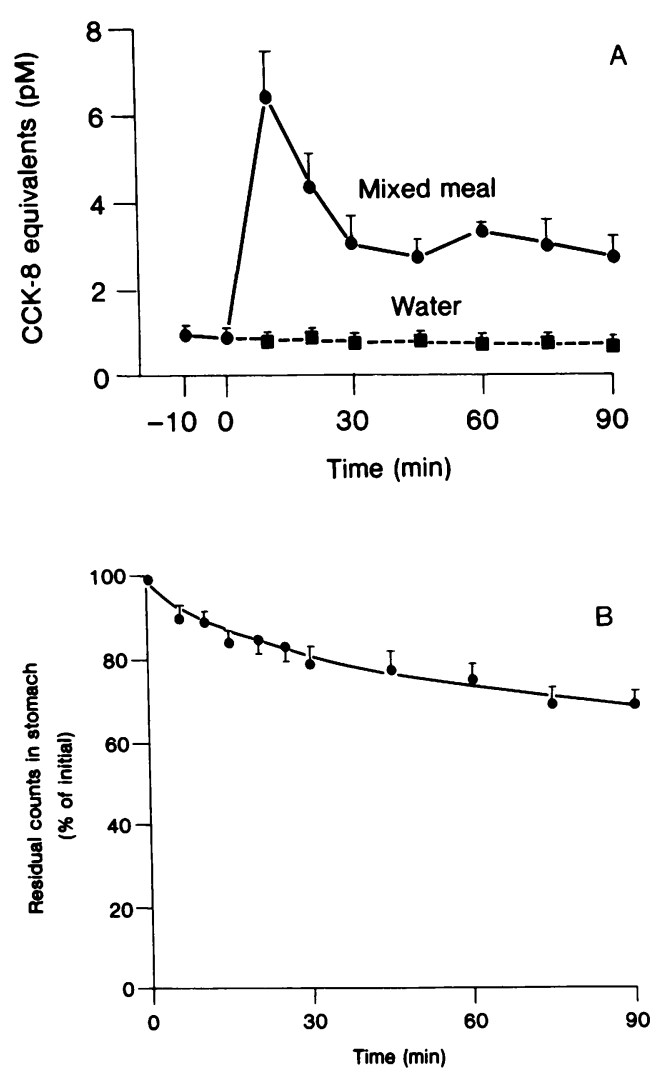

Figure 1. (A) Plasma CCK response to feeding. After an overnight fast five normal male subjects were fed a $400 \mathrm{ml} \mathrm{Tc}{ }^{99 \mathrm{~m}}$-sulfur colloid-labeled liquid meal. At the times indicated plasma was collected, extracted, and assayed for CCK bioactivity. Values are the mean \pm SEM. All postprandial values were statistically different from basal $(P$ $<0.05$ ). (B) Gastric emptying of a mixed liquid meal. Simultaneous with measurements of CCK after drinking a radiolabeled mixed liquid meal, gastric emptying was measured by scintigraphic scanning of the abdomen in each subject. The amount of radioactivity over the area of the stomach at each time point is expressed as percent of total activity at the initial time 0 . Each value is the mean \pm SEM of five subjects.

tric emptying of water measured. On separate days the same five subjects drank $400 \mathrm{ml}$ of $\mathrm{Tc}^{99 \mathrm{~m}}$-labeled water and had measurements of plasma CCK concentrations during intravenous administration of either saline or two doses of synthetic CCK8 (Fig. 2). Saline infusion had no effect on plasma CCK. However, with infusion of CCK-8, plasma CCK levels increased promptly within $10 \mathrm{~min}$ of beginning the infusion and remained constant for the period of study. Two separate doses of CCK were chosen for infusion in an attempt to span the physiologic range. The lower dose ( $12 \mathrm{pmol} / \mathrm{kg}$ per $\mathrm{h})$ of CCK-8 produced plasma levels of $3.4 \mathrm{pM}$ while the higher dose $(24 \mathrm{pmol} / \mathrm{kg}$ per h) resulted in plasma levels of $8.0 \mathrm{pM}$ (Table I).

Gastric emptying during CCK infusion. $10 \mathrm{~min}$ after beginning the infusion of saline or CCK, the subjects drank $400 \mathrm{ml}$ of $\mathrm{Tc}^{99 \mathrm{~m}}$-labeled water. At the time that measurements of plasma CCK were made, scintigraphic scans of the abdomen quantified the amount of radioactivity remaining in the stomach (Fig. 3). With infusion of saline, water emptied rapidly from the stomach. By 90 min only $7.1 \%$ of the original gastric contents remained in the stomach. Infusion of CCK-8 at a rate of $12 \mathrm{pmol} / \mathrm{kg}$ per $\mathrm{h}$, resulted in a delay in gastric emptying such that $30.0 \%$ of the original volume remained in the stomach at the conclusion of 


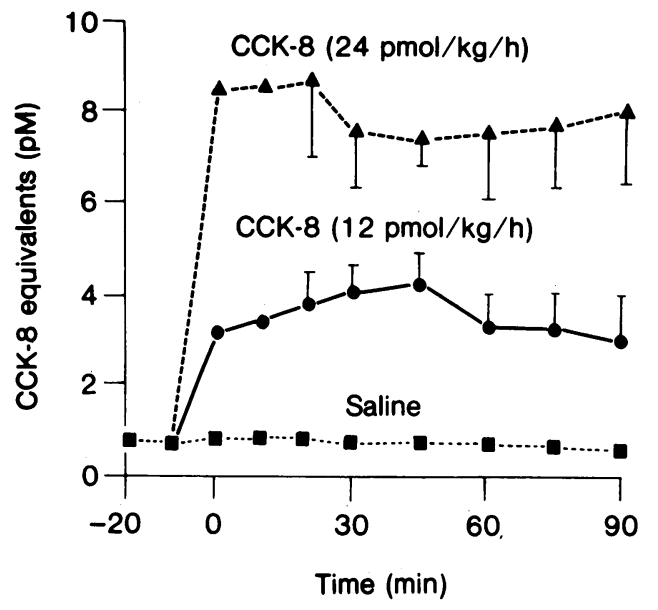

Figure 2. Plasma CCK levels after the infusion of CCK. On subsequent days each subject underwent intravenous infusion of either saline ( $\square$ ), low dose CCK (12 pmol/kg per h CCK-8, ๑), or high dose CCK (24 pmol/kg per h CCK-8, 4 ), while simultaneously drinking $400 \mathrm{ml}$ of $\mathrm{Tc}^{99 \mathrm{~m}}$-labeled water. Infusions began $10 \mathrm{~min}$ before ingestion of water and continued for $100 \mathrm{~min}$. Infusion doses for CCK were chosen to approximate those which occur normally after eating. Each value is the mean \pm SEM of five subjects studied.

the study. This effect was dose-dependent, as infusion of CCK8 at $24 \mathrm{pmol} / \mathrm{kg}$ per h caused a more profound delay in emptying such that $65.7 \%$ of the labeled water was retained in the stomach (Table I).

Delay of gastric emptying with infusion of CCK resulted in accumulation of residual volume in the body and fundus of the stomach (Fig. 4). This was appreciated in reviewing the scintiphotographs and was common to all subjects studied.

\section{Discussion}

Emptying of food from the stomach involves the complex integration of myogenic, neural, and humoral mechanisms. The

Table I. Relationship of Meal Content and Plasma CCK Levels to Gastric Emptying*

\begin{tabular}{|c|c|c|c|}
\hline Meal infusion & $\begin{array}{l}\text { Peak plasma } \\
\text { CCK level }\end{array}$ & $\begin{array}{l}\text { Average plasma } \\
\text { CCK level }\end{array}$ & $\begin{array}{l}\text { Residual volume in } \\
\text { stomach after } 90 \mathrm{~min} \\
\text { (percent of initial } \\
\text { volume) }\end{array}$ \\
\hline & $p M$ & $p M$ & \\
\hline Mixed saline meal & $6.5 \pm 1.0$ & $3.6 \pm 1.4$ & $68.3 \pm 3.6$ \\
\hline Water saline & $0.9 \pm 0.1$ & $0.8 \pm 0.1$ & $7.1 \pm 3.4$ \\
\hline \multicolumn{4}{|l|}{ Water CCK-8 (12 } \\
\hline pmol/kg per h) & $4.2 \pm 0.7$ & $3.4 \pm 0.4$ & $30.0 \pm 7.6$ \\
\hline \multicolumn{4}{|l|}{ Water CCK-8 (24 } \\
\hline pmol/kg per h) & $8.6 \pm 1.2$ & $8.0 \pm 0.5$ & $65.7 \pm 10.9$ \\
\hline
\end{tabular}

* Peak and average plasma CCK levels over the 90-min period of study attained under each condition (drinking a mixed meal, water, or water with CCK infusion) are shown in relation to the amount of radioactivity remaining in the stomach at the end of the study. Values are means \pm SEM for five subjects.

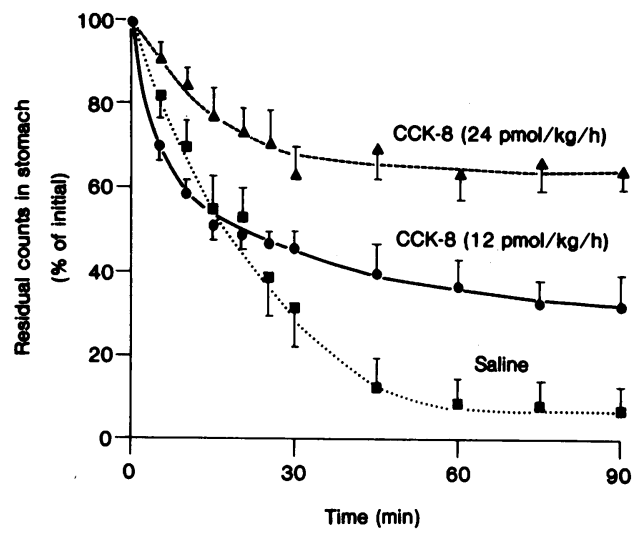

Figure 3. The effect of CCK infusion on the gastric emptying of water. 10 min after beginning the respective infusions shown in Fig. 2, each subject drank $400 \mathrm{ml}$ of water labeled with $100 \mathrm{uCi}$ of $\mathrm{Tc}^{99 \mathrm{~m}}$-sulfur colloid. The amount of radioactivity remaining in the stomach was determined by scintigraphic scanning of the stomach in each subject. Values are expressed as percent of total radioactivity in the stomach at the initial time 0 . Each value is the mean \pm SEM of five subjects.

properties of a meal which are the major determinants of the emptying of liquid from the stomach include: volume, $\mathrm{pH}$, osmolarity, and nutrient content (1-3, 6-8). Long-chain fatty acids (7) and amino acids (6) have been shown to be potent inhibitors of gastric emptying. Since these foods are potent stimulants of CCK release (9), it was important to determine whether CCK inhibits gastric emptying. Cholecystokinin had been shown to be a potent inhibitor of gastric emptying in dogs (14) and humans $(13,15)$ but its physiological relevance was unknown. The purpose of the present study, therefore, was to determine whether inhibition of gastric emptying by CCK occurs at physiological concentrations of the hormone

In the present study, we first fed subjects a mixed liquid meal and measured both plasma CCK levels and the rate of gastric emptying. ${ }^{2}$ Next we fed the same subjects water in the absence and presence of infusions of CCK. Water alone emptied from the stomach at a rate which was much faster than that of the liquid meal. CCK at physiological plasma concentrations altered the rate at which water emptied from the stomach. At a CCK infusion of $12 \mathrm{pmol} / \mathrm{kg}$ per $\mathrm{h}$ the resulting CCK levels were equivalent to the average CCK levels seen after the liquid meal and gastric emptying was significantly decreased. At a CCK infusion of $24 \mathrm{pmol} / \mathrm{kg}$ per $\mathrm{h}$ the resultant CCK levels were similar to the peak CCK levels seen after the meal, and gastric emptying was delayed to the same extent as seen with the liquid meal. These studies indicate, therefore, that physiological concentra-

2. This study employs a sensitive and specific bioassay for CCK that uses isolated rat pancreatic acini. The assay can readily measure fasting levels of CCK which are $1 \mathrm{pM}$ or less and the assay is not interfered with by either gastrin or other pancreatic secretagogues. Earlier radioimmunoassays for CCK were limited by their cross-reactivity with gastrin. Recently several antibodies have been produced which appear to be specific for CCK (29-32). Although the bioassay has the unique advantage of measuring biologically active $\mathrm{CCK}$, it has an inherent disadvantage in that only a limited number of samples can be assayed per day. It is likely, therefore, that routine radioimmunoassays for CCK may become available and could be used in conjunction with bioassays to measure circulating CCK levels. 

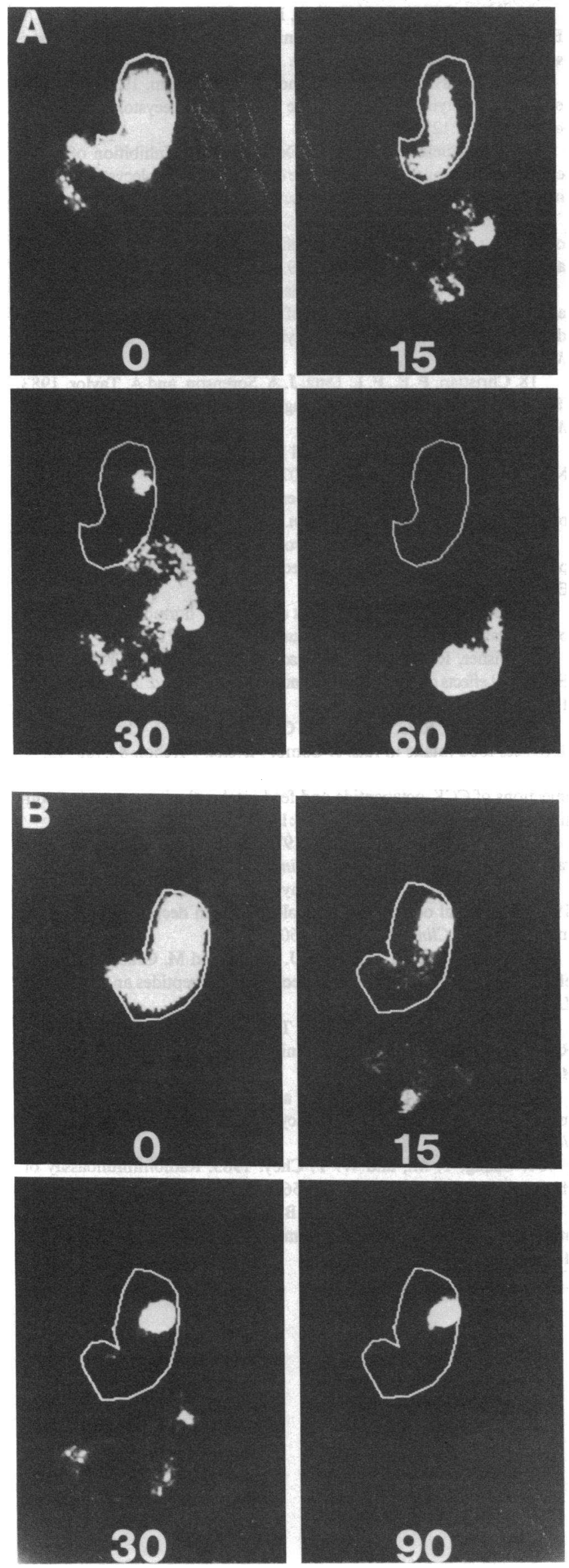

tions of CCK delay gastric emptying. Since other factors, as mentioned above, had been shown to also slow gastric emptying (1-6) it is likely that CCK is one of several physiological regulators of stomach motility.

Various studies in several species have indicated that administration of CCK inhibits gastric emptying (10, 12-15). Two of these studies attempted to assess whether the concentrations of CCK achieved were physiological, by either measuring pancreatic secretion (15) or pancreatic secretion plus gallbladder contraction (14). In the dog, Debas et al. concluded that the levels of CCK that inhibited gastric emptying were physiological (14). In contrast, Valenzuela and Defilippi concluded that the levels of CCK needed to inhibit gastric emptying in humans were supraphysiological. It is unfortunate that in both of these studies CCK was not measured directly either by bioassay or by radioimmunoassay.

A major control of the emptying of liquids from the stomach is the pressure gradient, generated primarily from contraction of the proximal stomach (20), that exists between the stomach and duodenum. In dogs, CCK decreases intragastric pressure by relaxing the proximal stomach $(11,20-22)$. It has also been demonstrated that CCK stimulates contraction of the pylorus $(11,23)$. Both of these actions of CCK on the stomach would delay gastric emptying. After CCK infusion in the present study, radioactive label accumulated in the body and fundus of the stomach. These findings in humans are consistent, therefore, with the concept that CCK delays gastric emptying by either relaxing the proximal stomach, contracting the pylorus, or both.

The finding that CCK slows gastric emptying is in concert with the role of $\mathrm{CCK}$ as a hormone that facilitates digestion. Concomitant with this effect on the stomach, there are effects of CCK both on contraction of the gallbladder and on stimulation of pancreatic exocrine secretion. Thus the multiple effects of CCK serve to coordinate the delivery of food into the intestine with the appearance of bile and pancreatic enzymes into that organ.

The effect of CCK on gastric emptying may constitute a feedback system whereby CCK regulates its own release. When food leaves the stomach and enters the duodenum CCK is released. The released CCK in turn inhibits gastric emptying. As a consequence of inhibiting gastric emptying less food is delivered to the duodenum and this subsequent CCK secretion is decreased.

Another potential consequence of the effect of CCK on the stomach is to regulate food intake. It is known that the administration of CCK to humans and experimental animals induces satiety (24-28). In animals, this effect occurs when CCK is given into either the blood or the central nervous system. While the effect of CCK on satiety may involve multiple mechanisms, it has been suggested that one mechanism whereby CCK induces satiety is to act directly on the stomach to induce gastric retention

Figure 4. The effect of CCK on the pattern of gastric emptying of water. Scintiphotographs show the patterns of gastric emptying of $\mathrm{Tc}^{99 \mathrm{~m}}$. labeled water in a subject during infusion of $(A)$ saline and $(B)$ low dose CCK (12 pmol/kg per $\mathrm{h}$ ). The outlined area was used for quantifying the amount of radioactivity in the stomach. Note in $A$ that the radioactivity emptied completely from the stomach by $60 \mathrm{~min}$ in contrast to $B$ where even at as late as 90 min a significant amount of radioactivity remained in the body and fundus of the stomach. This pattern was representative of the five subjects studied. 
(12). Both earlier studies and the present study indicate that CCK delays gastric emptying. It is possible, therefore, that this effect of CCK on gastric emptying may mediate, in part, the induction of satiety by CCK.

\section{Acknowledgments}

Expert technical support by Dolores Olson and Marilyn McFarland is appreciated. The authors thank Dr. I. D. Goldfine, Dr. K. A. Woeber, and Dr. J. H. Grendell for helpful suggestions in reviewing the manuscript.

This work was supported by National Institutes of Health grants AM32994 and AM01291; a grant from the Academic Senate of the University of California, San Francisco; and the Elise Stern Haas Research Fund of Mount Zion Hospital and Medical Center.

\section{References}

1. Hunt, J. N., and D. F. Stubbs. 1975. The volume and energy content of meals as determinants of gastric emptying. J. Physiol. (Lond.). 245:209-25.

2. Barker, G. R., G. M. Cochran, G. A. Corbett, J. F. Dufton, J. N. Hunt, and S. K. Roberts. 1978. Glucose, glycine, and diglycine in test meals as stimuli to a duodenal osmoreceptor slowing gastric emptying. J. Physiol. (Lond.). 283:341-6.

3. Hunt, J. N., and M. T. Knox. 1966. The slowing of gastric emptying by four strong acids and three week acids. J. Physiol. (Lond.). 154:25469.

4. Bateman, D. N. 1982. Effects of meal temperature and volume on the emptying of liquid from human stomach. J. Physiol. (Lond.). 331:461-467.

5. Hunt, J. N. 1983. Does calcium mediate slowing of gastric emptying by fat in humans? Am. J. Physiol. 244:G89-G94.

6. Stephens, J. R., R. F. Woolson, and A. R. Cooke. 1975. Effects of essential and non-essential amino acids on gastric emptying in the dog. Gastroenterology. 69:920-7.

7. Hunt, J. N., and M. T. Knox. 1968. A relation between the chain length of fatty acids and the slowing of gastric emptying. J. Physiol. (Lond.). 194:327-36.

8. Hunt, J. N. 1984. Regulation of gastric emptying by neurohumoral factors and by gastric and duodenal receptors. In Esophageal and Gastric Emptying. A. Dubois and D. O. Castell, editors. CRC Press, Boca Raton, Florida. 65-71.

9. Liddle, R. A., I. D. Goldfine, M. S. Rosen, R. A. Taplitz, and J. A. Williams. 1985. Cholecystokinin bioactivity in human plasma: molecular forms, responses to feeding, and relationship to gallbladder contraction. J. Clin. Invest. 75:1144-52.

10. Anika, M. S. 1982. Effects of cholecystokinin and caerulin on gastric emptying. Eur. J. Pharmacol. 85:195-9.

11. Yamagishi, T., and H. T. Debas. 1978. Cholecystokinin inhibits gastric emptying by acting on both proximal stomach and pylorus. Am. J. Physiol. 234:E375-E378.

12. Moran, T. H., and P. R. McHugh. 1982. Cholecystokinin suppresses food intake by inhibiting gastric emptying. Am. J. Physiol. 242: R491-R497.
13. Chey, W. Y., S. Hitanant, J. Hendricks, and S. H. Lorber. 1970. Effect of secretin and cholecystokinin on gastric emptying and gastric secretion in man. Gastroenterology. 58:820-7.

14. Debas, H. T., O. Farooq, and M. I. Grossman. 1975. Inhibition of gastric emptying is a physiologic action of cholecystokinin. Gastroenterology. 68:1211-7.

15. Valenzuela, J. E., and C. Defilippi. 1981. Inhibition of gastric emptying in humans by secretin, the octapeptide of cholecystokinin, and intraduodenal fat. Gastroenterology. 81:898-902.

16. Liddle, R. A., I. D. Goldfine, and J. A. Williams. 1984. Bioassay of plasma cholecystokinin in rats: effects of food, trypsin inhibitor, and alcohol. Gastroenterology. 87:542-9.

17. Van Deventer, G., J. Thomson, L. S. Graham, D. Thomasson, and J. H. Meyer. 1983. Validation of correction for errors in collimation during measurement of gastric emptying of nuclide-labeled mass. J. Nucl. Med. 24:187-96.

18. Christian, P. E., F. L. Datz, J. A. Sorenson, and A. Taylor. 1983. Technical factors in gastric emptying studies, teaching editorial. J. Nucl. Med. 24:264-8.

19. Winer, B. J. 1971. Statistical Principles in Experimental Design. McGraw-Hill, Inc. New York. 1-907.

20. Kelley, K. A. 1980. Gastric emptying of liquids and solids: roles of proximal and distal stomach. Am. J. Physiol. 239:G71-G76.

21. Strunz, U. T., and M. I. Grossman. 1978. Effect of intragastric pressure on gastric emptying and secretion. Am. J. Physiol. 235:E552E555.

22. Valenzuela, J. E. 1976. Effect of intestinal hormones and peptides on intragastirc pressure in dogs. Gastroenterology. 71:766-9.

23. Fisher, R. S., K. Phaosawasdi, G. Boden, and B. Kolts. 1978. Hormonal effects on the pyloric sphincter in man. Scand. J. Gastroenterol. 13 (Suppl 49):62.

24. Gibbs, J., R. C. Young, and G. P. Smith. 1973. Cholecystokinin decreases food intake in rats. J. Comp. Physiol. Psychol. 84:488-95.

25. Della-Fera, M. A., and C. A. Baile. 1980. Cerebral ventricular injections of CCK-octapeptide and feed intake: the importance of continued injection. Physiol. Behav. 24:1133-8.

26. Gibbs, J., and G. P. Smith. 1977. Cholecystokinin and satiety in rats and rhesus monkeys. Am. J. Clin. Nutr. 30:758.

27. Kissileff, H. R., F. X. Pi-Sunyer, J. Thornton, and G. P. Smith. 1981. C-terminal octapeptide of cholecystokinin decreases food intake in man. Am. J. Clin. Nutr. 34:154-60.

28. Morely, J. E., A. S. Levine, J. Kniep, and M. Grace. 1982. The effect of vagotomy on the satiety effects of neuropeptides and naloxone. Life Sci. 30:143-7.

29. Byrnes, D. L., L. Henderson, T. Borody, and J. F. Rehfeld. 1981. Radioimmunoassay of cholecystokinin in human plasma. Clin. Chim. Acta. 111:81-89.

30. Schafmayer, A., M. Werner, and H. D. Becker. 1982. Radioimmunological determination of cholecystokinin in tissue extracts. Digestion. 24:146-154.

31. Chang, T. M., and W. Y. Chey. 1983. Radioimmunoassay of cholecystokinin. Dig. Dis. Sci. 28:456-468.

32. Jansen, J. B. M. J., and C. B. H. W. Lamers. 1983. Radioimmunoassay of cholecystokinin in human tissue and plasma. Clin. Chim. Acta. 131:305-309. 